\title{
Vesico-cervical fistula following normal vaginal delivery: case report and management overview
}

\author{
Vijaya Koothan* \\ Department of Obstetrics and Gynecology, Arunai Medical College and Hospital Thiruvannamalai, Tamil Nadu, India
}

Received: 25 May 2021

Revised: 01 July 2021

Accepted: 02 July 2021

\section{*Correspondence:}

Vijaya Koothan,

E-mail: vijayakoothan7@gmail.com

Copyright: ( $)$ the author(s), publisher and licensee Medip Academy. This is an open-access article distributed under the terms of the Creative Commons Attribution Non-Commercial License, which permits unrestricted non-commercial use, distribution, and reproduction in any medium, provided the original work is properly cited.

\begin{abstract}
Vesico-uterine fistula is an uncommon pathological communication developing between the uterus or cervix and the urinary bladder especially in traumatic caesarean sections. We presented a case of vesico-cervical fistula who presented after 18 years of occurrence of fistula and successful repair. Clinical diagnosis may be delayed due to varied presentations and evaluation may require more than one modality of investigation. The choice of treatment surgical or conservative management depends on the location size and number of the fistula. Surgical outcomes of open laparotomy, laparoscopic and robotic surgery have been successful. Obstetric outcomes of post repair patients have had successful pregnancies.
\end{abstract}

Keywords: Youssef syndrome, Vesico-uterine fistulas, Vesico-cervical fistula

\section{INTRODUCTION}

Vesico-uterine fistula (VUF) is an uncommon pathological communication developing between the uterus or cervix and the urinary bladder. Caesarean section $(83 \%$ to $93 \%$ of cases) especially traumatic caesarean section. Youssef's syndrome classically presents as a triad of cyclical hematuria, amenorrhea and urinary continence, that is, there is no vaginal leakage of urine. Iatrogenic injury to the lower urinary tract is an uncommon $(0.1 \%$ to $0.3 \%)$ complication associated with caesarean delivery. Youssef first described 'Menouria' resulting from VUF in 1957. ${ }^{1}$ Jozwik et al in their series reported significant increase in incident of vesicouterine versus 58.3\% (14/24) from 1983 to $1994(\mathrm{p}=0.013)^{2,3}$

A classification of VUF based on the routes of menstrual flow has been proposed by Jo'zwik that divides VUF into three types- (a) type I, characterize by the triad of amenorrhea, menouria and complete continence of urine, known as Youssef's syndrome; (b) type II is associated with dual menstrual flow via both the bladder and vagina; and (c) type III is associated with normal vaginal menses and without menouria, which leads to delay in patient presenting to the health care provider. ${ }^{4,5}$.Amenorrhea, cyclic hematuria without urinary incontinence in combination with a history of LSCS, has been described as pathognomonic of VUF. ${ }^{6}$ The clinical presentation is often nonspecific and clinical findings may be elusive or, leading to considerable delay in diagnosis. ${ }^{67}$ Urinary incontinence may not be consistent finding in VUF because of a functional sphincter at the internal uterine os. Incontinence presents if the level of the VUF is at or below the internal os or if the os is incompetent. ${ }^{7}$

Spontaneous closure of VUF has been reported following catheterisation for two months. Conservative approach associated with hormonal suppression of menstruation, offers better results if the suppression is initiated soon after the diagnosis. ${ }^{8}$ Mode and outcome of surgery depends on size location and number of fistulae and expertise of the surgeon. 
VUF poses challenges in the clinical diagnosis owing to different presentation depending on the site of the fistulae and evaluation may require more than one modality of investigation. Judicious choice of patients for both conservative and surgical repair brings about desirable outcomes of treatment of fistula.

\section{CASE REPORT}

A 43 years old lady $\mathrm{P}_{2} \mathrm{~L}_{2}$ presents with complaints of increased frequency of urination especially in the night 10 times a day for 18 years. History was suggestive of menouria, urinary incontinence with change of posture. She delivered 2 babies by normal vaginal delivery. Patient had last child birth 18 years back. There was no history of prolonged labour, or instrumental delivery, she delivered average sized baby, underwent tubectomy on $3^{\text {rd }}$ post-natal day. Patient had history of urinary incontinence since $10^{\text {th }}$ post-natal day. No other significant gynaecological, past, personal history was elicited.

$\mathrm{O} / \mathrm{E}$ patient was afebrile, well built, well nourished, vital signs were stable, general examination and systemic examination was unremarkable. Per-abdominal examination revealed a uterus 18 weeks size irregular firm, non-tender, external genitalia normal, a gush of watery discharge was observed on per speculum examination, no fistula could be located per speculum. Bimanual examination revealed 18 to 20 weeks irregular firm nontender uterus.

Examination with 3 tampon tests was positive, with upper tampon in the vicinity of the fornices stained methylene blue. On investigation for pre-operative evaluation revealed to be newly diagnosed with diabetic mellitus with fasting blood sugar- $171 \mathrm{mg} \%$, and postprandial blood sugar $301 \mathrm{~g} \%$. Urine examination suggestive of haematuria and albuminuria. Urine culture and sensitivity suggestive of E. coli sensitive to imipenum

Cystoscopy on 21/11/06 revealed, bladder smooth walled, fistula in the dome of bladder, bilateral ureteric orifices were normal, ureteric catheterization done.

\section{Impression}

Para 2 live 2 with Youssef syndrome- VUF with type II diabetes mellitus.

Patient was posted for laparotomy, underwent total abdominal hysterectomy with bilateral sylphingoopherectomy, with vesico-cervical fistula repair. Intra-operative findings: uterus 20 weeks intramural fibroid, $0.5 \mathrm{~cm}$, vesico-cervical fistula. On methylene blue instillation into the urinary bladder, the dye was seen seeping through the cervix, demonstrating the vesicocervical fistula. Tubes and ovaries were normal. HPE of TAH-BSO chronic cervicitis, endometritis, leomyoma. Post-operative period was uneventful, patient was symptom free.

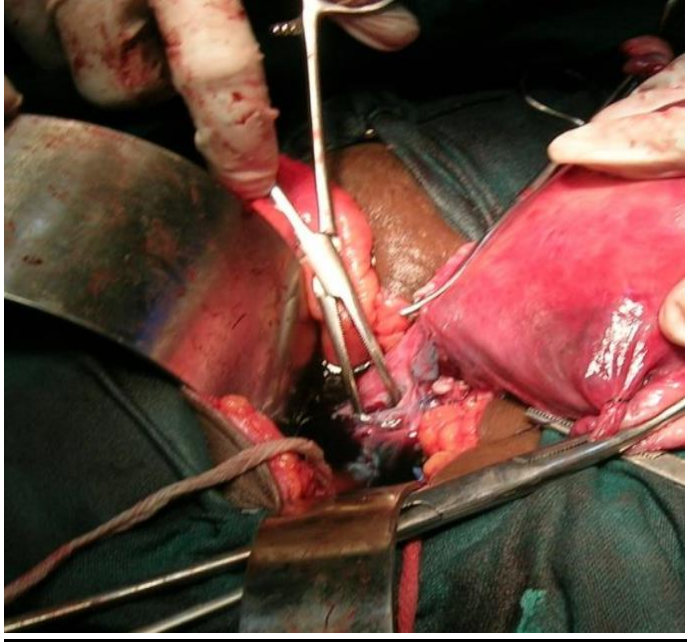

Figure 1: Showing methylene blue flowing from bladder through a small fistula in the cervix.

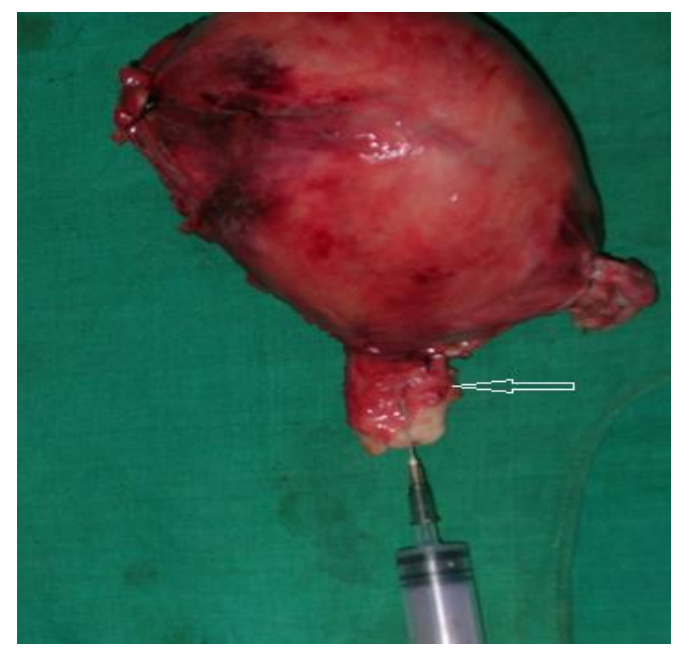

Figure 2: TAH-BSO specimen with myoma and showing the site of fistula on the cervix below the level of internal as shown by the arrow.

\section{DISCUSSION}

\section{Etiology}

The VUF has been reported following high vaginal forceps delivery, external cephalic version, curettage or manual removal of the placenta, placenta percreta, myomectomy, uterine rupture due to obstructed labour, uterine artery embolization, perforation of an intrauterine device, and brachytherapy for carcinoma of cervix. But the single most common cause for VUF is following a traumatic caesarean section. Various other etiologies have been reported in the literature following placenta percreta, manual removal of placenta after vaginal birth after caesarean, excision of Gartner-cyst, anterior colporrhaphy, endometrial ablation, myomectomy, tuberculosis, actinomycosis and cervical circular procedures. The hypotheses proposed are undetected bladder rupture during caesarean section in the $2^{\text {nd }}$ stage, inadvertent inclusion of bladder in the uterine 
suture, insufficient blood supply to the base of bladder due to multiple dissections following repeat sections. ${ }^{9-11}$

Youssef proposed a sphincter/valve mechanism, exerted by uterine isthmus, to explain the absence of urinary incontinence in this condition. During most part of the menstrual cycle, the intrauterine pressure is greater than the intra-vesical pressure. Only during a very brief period of the cycle, the intra-vesical pressure overcomes intrauterine pressure when the woman is voiding. This mechanism explains the intermittent incontinence related by some women. ${ }^{12}$

\section{Diagnosis}

The first step in the diagnosis is, of course, a suggestive clinical presentation. The absence of urinary incontinence could be misleading in the diagnosis and delay of diagnosis. Ugurlucan et al reported a case of delayed presentation of VUF in a 55-years-old woman who presenting with urinary incontinence 30 years after a caesarean section. ${ }^{13}$

The confirmation of the diagnosis is by cystoscopy after the injection of dye in the bladder, cystography, intravenous pyelography, hysterography and sonographies, MRI have been used in the investigation. Sometimes more than one modality of investigation may be required to demonstrate the fistula. ${ }^{14,15}$

Trans-abdominal ultrasound evaluation of urogenital fistulae, poorly identified the fistula with a sensitivity in as low as $29 \%$. Abou et al, reported in a small case series $100 \%$ sensitivity with MRI in the diagnosis of VUF. ${ }^{16}$.

Trans-urethral or trans-uterine catheterization and instillation of methylene blue may detect the fistula. But this test can be negative in fistulae with long and tortuous tracts Tancer in his review reports hysterography was the most reliable diagnostic technique. Intravenous urography can show the fistula when contrast medium enters the vagina, but distinguishing vesico-vaginal and VUF may be difficult. Hysterography and cystoscopy remain the 'gold standard' in the diagnosis ${ }^{17}$.

Additionally, it is recommended that the physician defines precisely the size and course and number of fistulae, because of this, it is usual to perform two or more investigation modality during the evaluation. Intravenous pyelography to allow view of the entire urinary tract. There are recent reports about diagnosis with computed tomography and nuclear magnetic resonance imaging are reported although not mandatory. ${ }^{18,19}$ Magnetic resonance imaging (MRI) of the pelvis reveals fluid-filled fistulous communication between the urinary bladder.

\section{Treatment}

Treatment options include expectant management with long-term bladder catheterization, medical treatment, and surgery. Oral contraceptives, progestational agents, and gonadotropin releasing hormone analogs have been used to induce amenorrhea and promote healing of the fistula.

Surgery is the definitive method of treatment transabdominally, endoscopically, or robotically. The transvaginal approach is may not be preferred because of the higher location and complexity of the fistulae.

The proposed treatments for VUF are: (1) surgical resection with interposition of- (a) through abdominal open surgery momentum layer between the uterus and (b) the bladder to diminish the risk of recurrence of the fistula is recommended; (2) laparoscopic correction; (3) vaginal approach; (4) robotic-assisted surgery. In the surgical treatment, the choice of the route, namely open abdominal, laparoscopic, transvaginal or robotic, depends on the location of the fistula and, on the experience of the team in non-conventional routes (laparoscopic, transvaginal or robotic). Hemal et al reports successful robotic repair of even complex vesicouterine fistulae in 8 out of 8 women. ${ }^{20}$

The best results reported are with the open abdominal route. Although laparoscopy, vaginal and robotic approaches yield good results. Molina et al 50 reported cures with fulguration of the fistulous tract through cystoscopy in a small fistula. ${ }^{21}$

There are many reports of conservative treatment and spontaneous cure. Jozwik et al their review, found 29 cases of spontaneous resolution, $5.1 \%$ of cases (41/796). When subjected to hormonal suppression of menstruation with progestogens or combined oral contraceptives, 8/9 had their fistulas cured $(88.9 \%)$. In the remaining 787 women without hormonal suppression, 33 were cured (4.2\%). with the Chi square test and concluded that the difference was statistically significant $(\mathrm{p}<0.001)$.

Conservative approach associated with hormonal suppression of menstruation, offers better results if the suppression is initiated soon after the diagnosis. The presence of urinary incontinence is suggesting poor prognosis for spontaneous closure of fistula in which case conservative approach is the option, the patient should remain with continuous bladder drainage for at least three months. If the surgical correction is not performed in the first few days after the caesarean section, it is recommended to postpone it for two to three months, to allow for involution and complete resolution of the inflammatory process related to scar formation. Many studies suggest successful fertility and obstetric outcomes among women with VUF post repair. Lotocki et al reported among 4 women who attempted pregnancy out of 16 women who underwent fistula repair had successful pregnancies. $^{22}$ Many women were anxious about recurrence on having another pregnancy after repair and therefore did not plan pregnancy. Rao et al reported 3 successful pregnancies out of 8 women who underwent fistula repair. Dein et al reported 5 out of 22 women had successful obstetrics outcome after VUF repair but the 
actual number of women who intended pregnancy is not mentioned. Generally, repeat caesarean section is advised post repair in many studies but no studies report attempt on vaginal delivery. ${ }^{21}$

\section{CONCLUSION}

VUF poses challenges in the clinical diagnosis owing to different presentation depending on the site of the fistulae and evaluation may require more than one modality of investigation. Judicious choice of patients for both conservative and surgical repair brings about desirable outcomes of treatment of fistula. Obstetric outcome has been reported to be successful in many women who have attempted another pregnancy.

\section{ACKNOWLEDGMENTS}

Authors would like to thank Miss. Kavitha, junior assistant assisted in typing the manuscript.

Funding: No funding sources

Conflict of interest: None declared

Ethical approval: Not required

\section{REFERENCES}

1. Youssef AF. Menouria following lower segment cesarean section; a syndrome. Am J Obstet Gynecol. 1957;73(4):759-67.

2. Jozwik M, Jozwik M. Spontaneous closure of vesicouterine fistula. Account for effective hormonal treatment. Urol Int. 1999;62(3):183-7.

3. Jozwik M, Jozwik M, Lotocki W. Vesicouterine fistula--an analysis of 24 cases from Poland. Int $\mathrm{J}$ Gynaecol Obstet. 1997;57(2):169-72.

4. Jozwik M, Jozwik M. Clinical classification of vesicouterine fistula. Int $\mathbf{J}$ Gynaecol Obstet. 2000;70(3):353-7.

5. Bhattacharjee S, Kohli UA, Soodb A, Tripathy S, Mihir. Vesicouterine fistula: Youssef's syndrome. Medical J armed forces India. 2015;71:175-7.

6. Birge O, Ozbey EG, Erkan MM, Arslan D, Kayar I. Youssef's Syndrome following Cesarean Section. Case Rep Obstet Gynecol. 2015;2015:605325.

7. Yip SK, Leung TY. Vesicouterine fistula: an updated review. Int Urogynecol J Pelvic Floor Dysfunct. 1998;9(5):252-6.

8. Rao MP, Dwivedi US, Datta B, Vyas N, Nandy PR, Trivedi S, Singh PB. Post caesarean vesicouterine fistulae- Youssef syndrome: our experience and review of published work. ANZ J Surg. 2006;76(4):243-5.

9. Dein B, Tabey N, Hefnawy A, Osman Y, Soliman S, Shaaban AA. Diagnosis, treatment and need for hysterectomy in management of postcaesarean section vesicouterine fistula. Scand J Urol. 2014;48(5):460-5.

10. Ikechebelu JI, Ugboaja JO, Okeke CF. Post-cesarean vesicouterine fistula (Youssef syndrome): report of two cases. J Obstet Gynaecol Res. 2011;37(7):912-5.

11. Czerwinski F, Pilarczyk K, Kozik W. Vascularization of the posterior wall of the urinary bladder in women after past cesarean section in light of microangiographic studies. Ginekol Pol. 1992;63(8):398-403.

12. Machado RA, Machado LC, Lourenco LL. Vesicouterine Fistula (Youssef Syndrome): Case Report and Literature Review. SciElo Brazil. 2018;4(9):563-9.

13. Ugurlucan FG, Bastu E, Bakir B, Yalcin O. Vesicouterine fistula presenting with urinary incontinence 30 years after primary Cesarean: Case report and review of the literature. Can Urol Assoc J. 2014;8(1-2):48-50.

14. Smayra T, Ghossain MA, Buy JN, Moukarzel M, Jacob D, Truc JB. Vesicouterine fistulas: imaging findings in three cases. AJR Am J Roentgenol. 2005;184(1):139-42.

15. Benchekroun A, Lachkar A, Soumana A, Farih MH, Belahnech Z, Marzouk M, Faik M. Vesico-uterine fistulas. Report of 30 cases. Ann Urol. 1999;33(2):759.

16. Ghar ME, Assmy AM, Refaie HF, Diasty TA. Radiological diagnosis of vesicouterine fistula: role of magnetic resonance imaging. J Magn Reson Imaging. 2012;36(2):438-42.

17. Tancer ML. Vesicouterine fistula--a review. Obstet Gynecol Surv. 1986;41(12):743-53.

18. Singh V, Sinha RJ, Mehrotra S. Primary menouria due to a congenital vesico-vaginal fistula with distal vaginal agenesis: a rarity. Int Urogynecol J. 2011;22(8):1031-3.

19. Purkait, Mehratra S, Sinha RJ, Bhaskar V, Singh V. Long-term outcome of laproscoic vesicouterine Fistula repair: Experience from a tertiary referral centre. Turk J Urol. 2017;43(4):512-6.

20. Perveen K, Gupta R, Al-Badr A, Hemal AK. Robotassisted laparoscopic repair of rare post-cesarean section vesicocervical and vesicouterine fistula: a case series of a novel technique. Urology. 2012;80(2):47782.

21. Molina LR, Lynne CM, Politano VA. Treatment of vesicouterine fistula by fulguration. $\mathrm{J}$ Urol. 1989;141(6):1422-3.

22. Lotocki W. Jozwik M. Jozwik M prognosisof fertility after surgical closure of vesicouterine fistula. Eur $\mathbf{J}$ Obstet Gynecol Reprod Biol 1996;64(01):87-90.

Cite this article as: Koothan V. Vesico-cervical fistula following normal vaginal delivery: case report and management overview. Int J Reprod Contracept Obstet Gynecol 2021;10:3232-5. 